\title{
Is styrene planar in liquid phases?
}

\author{
Giorgio Celebre,${ }^{\text {a) }}$ Giuseppina De Luca, ${ }^{\text {b) }}$ Marcello Longeri, ${ }^{c)}$ and Giuseppe Pileio ${ }^{\text {d) }}$ \\ Dipartimento di Chimica, Università della Calabria, Rende (CS), 87036, Italy \\ James W. Emsleye) \\ Chemistry Department, University of Southampton, Southampton SO17 1BJ, United Kingdom
}

(Received 11 December 2003; accepted 16 January 2004)

\begin{abstract}
The proton NMR spectra of two ${ }^{13} \mathrm{C}$-labeled isotopomers of styrene dissolved in two liquid crystalline solvents have been obtained and analyzed to yield four sets each of 24 dipolar couplings. These couplings were then used to investigate the structure of the ring and the ene fragments of the molecule, and the position of the maximum, $\phi_{0}$, in the ring-ene bond rotational probability distribution. To do this, the effect on the dipolar couplings of small-amplitude vibrational motion was taken into account using vibrational wave functions calculated by molecular orbital and density functional methods. It is concluded that the NMR data are consistent with the ring fragment, averaged over the ring-ene rotation, planar, while the ene fragment is not. The value of $\phi_{0}$ is found to be $18.0^{\circ} \pm 0.2^{\circ}$ for the two solutions, compared with a value of $27^{\circ}$ calculated by the molecular method MP2/6-31G*. (C) 2004 American Institute of Physics. [DOI: 10.1063/1.1668636]
\end{abstract}

\section{INTRODUCTION}

The most interesting aspect of the structure of the styrene molecule, shown in Fig. 1, is whether the olefin and aromatic parts are coplanar in the lowest-energy form. The two groups rotate relative to each other in liquid and gaseous samples, and so a related question concerns the shape of the rotational potential $V(\phi)$ about the ring-olefin bond.

There do not appear to have been any structure determinations on solid styrene; freezing a sample of styrene usually leads to polymerization. There have been several theoretical calculations on styrene, using methods available in the Gaussian suite of programs. ${ }^{1}$ The latest studies using HF/6-31G* (Ref. 2) and MP2/6-31G* (Ref. 3) find a nonplanar structure with a minimum in $V(\phi)$ in the range $16^{\circ}-27^{\circ}$. However, calculations by the density functional theory (DFT) method B3LYP/6-311+ $+\mathrm{G}^{* *}$ (Ref. 4) find a planar ground-state structure; moreover, in the same paper, the authors claim B3LYP/6-311++ $\mathrm{G}^{* *}$ to be the best method for predicting vibrational force fields. The calculations refer to an isolated molecule and are most relevant for comparison with experimental studies on gaseous samples. There has not been a full structure determination from microwave spectroscopy, but studies of para-substituted styrenes ${ }^{5,6}$ concluded that these molecules are planar. A recent electron diffraction study concluded that the data were consistent with either a planar form or one with the nonplanar structure calculated with MP2/6-31G* (Ref. 3).

Structural studies by NMR spectroscopy are greatly enhanced when the molecules under investigation are in a sample which is partially oriented. In this case the spectra are

\footnotetext{
a)Electronic mail: giorgio.celebre@unical.it

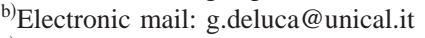

c) Author to whom correspondence should be addressed.

Fax: (39)0984492044. Electronic mail: m.longeri@unical.it

d)Electronic mail: g.pileio@unical.it

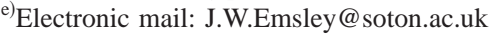

affected by anisotropic interactions such as the dipolar coupling $D_{i j}$ and quadrupolar splitting $\Delta \nu_{i}$ (Refs. 7-9). The partial orientation may be created by application of a strong magnetic field $(>9 \mathrm{~T}),{ }^{10}$ and perdeuterated styrene was studied in this way by Facchini et al., ${ }^{11}$ who measured the six quadrupolar splittings and concluded that these are consistent with a nonplanar styrene with a minimum in $V(\phi)$ at $16.5^{\circ}$. The orientational order induced by an applied magnetic field is very small, with order parameters of about $10^{-5}$ (Ref. 12) for fields of $9 \mathrm{~T}$, and the dipolar couplings in molecules such as styrene are of the order of $1 \mathrm{~Hz}$, and have not been measured for this molecule. Dipolar couplings are, however, a rich source of structural information, in part because there are potentially a large number of these, but also because they are directly related to the magnitude and orientation of the internuclear vectors connecting the interacting nuclei. A set of 18 interproton couplings and 6 quadrupolar splittings has been reported for a sample of styrene dissolved in a thermotropic, nematic, liquid crystalline solvent by Emsley and Longeri, ${ }^{13}$ who interpretated this data set in terms of a planar molecule. However, their interpretation and that of Facchini et al. ${ }^{11}$ relied on the assumption that the orientational order is independent of the bond rotation angle $\phi$. This assumption has been shown to be incorrect. ${ }^{14,15}$ More recently Ramadan et al. ${ }^{16}$ have demonstrated how multiple quantum NMR experiments can aid in the analysis of the proton spectrum of styrene dissolved in a nematic solvent. They interpreted their data in terms of two planar fragments inclined at an angle of $16^{\circ}$ and without considering vibrational motion.

There are insufficient data from the deuterium spectrum of perdeuteriated styrene to obtain the form of $V(\phi)$ while allowing for the dependence of the orientational order on $\phi$, but this is not the case for the dipolar couplings. It was decided to revisit the question of whether dipolar couplings can reveal the nature of $V(\phi)$ in styrene, and to increase the number of couplings available for this conformational analy- 


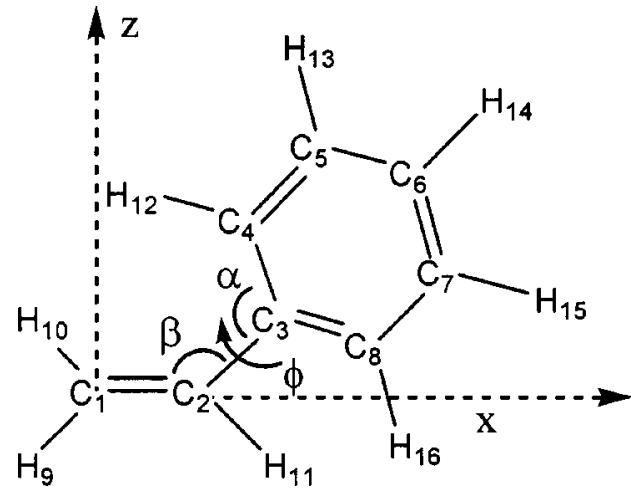

FIG. 1. Structure and labeling system for styrene.

sis, it was decided to record proton spectra of samples of styrene labeled with ${ }^{13} \mathrm{C}$ at positions $\mathrm{C} 1$ or $\mathrm{C} 2$ and dissolved in two nematic liquid crystalline solvents ZLI 1132 and I35. This increases the data set for each isotopomer in each solvent to 24 and allows the structures of the ring and olefinic fragments to be determined without needing to know the form of $V(\phi)$. The effects on the dipolar couplings of smallamplitude vibrational motion are calculated by $a b$ initio methods, and these are found to be particularly large, and hence important, for the dipolar couplings between nuclei in the olefinic fragment. The effect of orientation-conformation coupling is also included when investigating the form of $V(\phi)$. Taking into account the structures of the two fragments, allowing for vibrational motion and the coupling between orientation and conformation, has the desired effect of allowing a definitive statement to be made about the planarity of styrene in a condensed, liquid medium.

\section{EXPERIMENT}

The samples of ${ }^{13} \mathrm{C}$ labeled styrene were obtained from Isotech, and the liquid crystalline solvents were purchased from Merck (Darmstadt). The samples for recording the NMR spectra were each $10 \%$ by weight of the styrene and the proton spectra were recorded at $300 \mathrm{~K}$ on a Bruker AC 300 spectrometer working at 7.04 T. Figure 2 shows the proton spectrum of ${ }^{13} \mathrm{C} 2$-styrene dissolved in $\mathrm{I} 35$ and is typical of those obtained on the four samples. The free induction decays were stored in $32 \mathrm{k}$ words of computer memory, giving a precision on measuring peak positions of $0.76 \mathrm{~Hz}$.

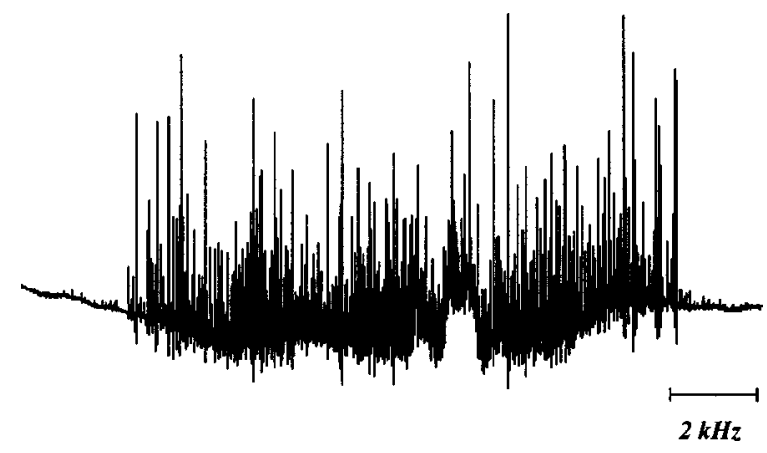

FIG. 2. $300-\mathrm{MHz}{ }^{1} \mathrm{H}$ spectrum of ${ }^{13} \mathrm{C} 2$-styrene dissolved in the nematic solvent $\mathrm{I} 35$ at $300 \mathrm{~K}$.

\section{ANALYSIS OF SPECTRA}

The analysis of the proton spectra of ${ }^{13} \mathrm{C} 2$-styrene and ${ }^{13} \mathrm{C} 1$-styrene dissolved in two liquid crystalline solvents proceeded in stages. The first stage was to analyze the proton spectra of samples of styrene dissolved in the same solvents at nearly identical concentrations. It was possible then to predict reasonable values of the $\mathrm{C}-\mathrm{H}$ couplings, and these, together with the interproton couplings obtained from the analysis of the styrene samples, gave a good starting set for an iterative analysis of the spectra of the two isotopomers using the program ARCANA. ${ }^{17}$ The results are given in Table I.

\section{RESULTS AND DISCUSSION}

If the phenyl ring and ene fragment maintain a fixed structure as they rotate relative to each other, then dipolar couplings within each of these fragments, $D_{i j}^{R}$ (equil) and $D_{i j}^{E}$ (equil), are related to the positions of the interacting nuclei and the local order parameters, $S_{a b}^{R}$ and $S_{a b}^{E}$, where $a$ and $b$ refer to axes fixed in each of the fragments. Thus,

$$
\begin{aligned}
D_{i j}^{R / E}(\text { equil })= & -\left(K_{i j} / r_{i j}^{3}\right)\left[S_{a a}^{R / E}\left(3 \cos ^{2} \theta_{i j a}-1\right)\right. \\
& +\left(S_{b b}^{R / E}-S_{c c}^{R / E}\right)\left(\cos ^{2} \theta_{i j b}-\cos ^{2} \theta_{i j c}\right) \\
& +4 S_{a b}^{R / E} \cos \theta_{i j a} \cos \theta_{i j b} \\
& +4 S_{a c}^{R / E} \cos \theta_{i j a} \cos \theta_{i j c} \\
& \left.+4 S_{b c}^{R / E} \cos \theta_{i j b} \cos \theta_{i j c}\right]
\end{aligned}
$$

with

$$
K_{i j}=\frac{\mu_{0} \hbar \gamma_{i} \gamma_{j}}{16 \pi^{2}} .
$$

The angles $\theta_{i j a}$, etc., are between the vector $r_{i j}$ and the axes $a b c$. The couplings $D_{i j}^{R / E}$ (equil) are for a molecule in a fixed equilibrium structure, while the observed couplings $D_{i j}^{R / E}$ (obs) are averages over vibrational motion. The two are related by

$$
D_{i j}^{R / E}(\text { obs })=D_{i j}^{R / E}(\text { equil })+D_{i j}^{R / E}(\text { vib }) .
$$

\section{A. Calculation of $D_{i j}^{R / E}$ (vib)}

The vibrational, correction term $D_{i j}^{R / E}$ (vib) has been calculated for styrene by assuming that this motion is harmonic, using the expression ${ }^{20}$

$$
D_{i j}^{R / E}(\mathrm{vib})=-K_{i j} \sum_{\alpha \beta} S_{\alpha \beta} \Phi_{\alpha \beta, i j}^{h},
$$

where

$$
\begin{aligned}
\Phi_{\alpha \beta, i j}^{h}= & {\left[C_{\alpha \beta}^{i j}-5 \sum_{\gamma} \cos \theta_{\gamma}\left(C_{\alpha \gamma}^{i j} \cos \theta_{\beta}+C_{\beta \gamma}^{i j} \cos \theta_{\alpha}\right)\right.} \\
& +\frac{5}{2} \cos \theta_{\alpha} \cos \theta_{\beta} \sum_{\gamma \delta} C_{\gamma \delta}^{i j} \\
& \left.\times\left(7 \cos \theta_{\gamma} \cos \theta_{\delta}-\delta_{\gamma \delta}\right)\right] r_{i j}^{-5},
\end{aligned}
$$


TABLE I. Dipolar couplings $D_{i j}$, scalar couplings $J_{i j}$, both in $\mathrm{Hz}$, and chemical shifts $\Delta \nu_{i j}=\nu_{I}-\nu_{j}$, in $\mathrm{Hz}$, obtained for samples, denoted as ZLI 1132 (C1) or (C2) and $\mathrm{I} 35$ (C1) or (C2), of ${ }^{13} \mathrm{C} 1$-styrene and ${ }^{13} \mathrm{C} 2$ - styrene dissolved in the nematic liquid crystalline solvents ZLI 1132 and I35 at $300 \mathrm{~K}$.

\begin{tabular}{|c|c|c|c|c|c|}
\hline & & ZLI 1132 (C2) & ZLI 1132 (C1) & I35 (C2) & I35 (C1) \\
\hline$i, j$ & $J_{i j}{ }^{\mathrm{a}}(\mathrm{Hz})$ & \multicolumn{4}{|c|}{$D_{i j}(\mathrm{~Hz})$} \\
\hline 1,9 & 160.00 & & $-6332.55 \pm 0.14$ & & $-6436.01 \pm 0.10$ \\
\hline 1,10 & 154.00 & & $886.84 \pm 0.21$ & & $1617.10 \pm 0.12$ \\
\hline 1,11 & -0.97 & & $-12.54 \pm 0.16$ & & $60.12 \pm 0.12$ \\
\hline 1,12 & & & $-177.27 \pm 0.13$ & & $-170.88 \pm 0.10$ \\
\hline 1,13 & & & $-51.64 \pm 0.15$ & & $-52.29 \pm 0.11$ \\
\hline 1,14 & & & $-40.24 \pm 0.12$ & & $-41.76 \pm 0.11$ \\
\hline 2,9 & & $-966.43 \pm 0.10$ & & $-1036.08 \pm 0.11$ & \\
\hline 2,10 & -3.18 & $-29.43 \pm 0.12$ & & $40.26 \pm 0.11$ & \\
\hline 2,11 & 153.20 & $661.18 \pm 0.11$ & & $1465.10 \pm 0.11$ & \\
\hline 2,12 & -0.72 & $-169.54 \pm 0.07$ & & $-148.90 \pm 0.09$ & \\
\hline 2,13 & & $-74.40 \pm 0.08$ & & $-76.71 \pm 0.10$ & \\
\hline 2,14 & & $-61.89 \pm 0.07$ & & $-65.34 \pm 0.08$ & \\
\hline 9,10 & 1.05 & $-1991.78 \pm 0.09$ & $-1934.65 \pm 0.16$ & $-1684.97 \pm 0.09$ & $-1597.16 \pm 0.09$ \\
\hline 9,11 & 10.90 & $-1398.23 \pm 0.11$ & $-1364.80 \pm 0.13$ & $-1424.42 \pm 0.09$ & $-1353.05 \pm 0.09$ \\
\hline 9,12 & & $-401.60 \pm 0.06$ & $-392.14 \pm 0.11$ & $-412.73 \pm 0.09$ & $-391.94 \pm 0.08$ \\
\hline 9,13 & & $-132.74 \pm 0.06$ & $-129.01 \pm 0.10$ & $-139.32 \pm 0.08$ & $-132.19 \pm 0.08$ \\
\hline 9,14 & & $-102.05 \pm 0.08$ & $-99.87 \pm 0.12$ & $-109.05 \pm 0.08$ & $-103.46 \pm 0.07$ \\
\hline 10,11 & 17.50 & $134.61 \pm 0.10$ & $133.25 \pm 0.14$ & $263.04 \pm 0.12$ & $248.80 \pm 0.11$ \\
\hline 10,12 & & $-1458.79 \pm 0.08$ & $-1422.97 \pm 0.17$ & $-1540.57 \pm 0.08$ & $-1460.93 \pm 0.11$ \\
\hline 10,13 & & $-247.78 \pm 0.08$ & $-241.17 \pm 0.15$ & $-261.51 \pm 0.10$ & $-248.12 \pm 0.11$ \\
\hline 10,14 & & $-169.04 \pm 0.10$ & $-165.32 \pm 0.13$ & $-181.09 \pm 0.09$ & $-171.86 \pm 0.08$ \\
\hline 11,12 & & $-1296.45 \pm 0.09$ & $-1263.32 \pm 0.14$ & $-1345.48 \pm 0.10$ & $-1275.66 \pm 0.11$ \\
\hline 11,13 & & $-239.70 \pm 0.09$ & $-234.54 \pm 0.14$ & $-247.78 \pm 0.10$ & $-235.34 \pm 0.12$ \\
\hline 11,14 & & $-166.28 \pm 0.08$ & $-162.24 \pm 0.12$ & $-172.97 \pm 0.08$ & $-163.67 \pm 0.09$ \\
\hline 12,13 & 8.00 & $-2524.93 \pm 0.04$ & $-2463.03 \pm 0.08$ & $-2676.76 \pm 0.07$ & $-2539.05 \pm 0.06$ \\
\hline 12,14 & 2.00 & $-355.11 \pm 0.06$ & $-346.64 \pm 0.11$ & $-363.39 \pm 0.07$ & $-344.80 \pm 0.07$ \\
\hline 12,15 & & $-67.42 \pm 0.05$ & $-65.58 \pm 0.09$ & $-46.80 \pm 0.06$ & $-44.55 \pm 0.06$ \\
\hline 12,16 & 2.00 & $21.75 \pm 0.10$ & $21.65 \pm 0.15$ & $72.48 \pm 0.14$ & $68.03 \pm 0.12$ \\
\hline 13,14 & 6.00 & $-530.96 \pm 0.06$ & $-512.26 \pm 0.11$ & $-378.80 \pm 0.08$ & $-361.37 \pm 0.08$ \\
\hline \multirow[t]{2}{*}{13,15} & 2.00 & $20.76 \pm 0.10$ & $21.59 \pm 0.17$ & $71.55 \pm 0.15$ & $68.11 \pm 0.12$ \\
\hline & \multicolumn{2}{|c|}{$\Delta \nu_{i j}(\mathrm{~Hz})$} & & & \\
\hline $9-10$ & \multicolumn{2}{|c|}{$-592.17 \pm 0.08$} & $-592.26 \pm 0.15$ & $-616.52 \pm 0.09$ & $-615.17 \pm 0.09$ \\
\hline $10-11$ & \multicolumn{2}{|c|}{$-420.98 \pm 0.08$} & $-422.58 \pm 0.13$ & $-446.17 \pm 0.09$ & $-447.25 \pm 0.10$ \\
\hline $11-12$ & \multicolumn{2}{|c|}{$-269.73 \pm 0.08$} & $-267.27 \pm 0.14$ & $-291.91 \pm 0.09$ & $-286.07 \pm 0.10$ \\
\hline $12-14$ & \multicolumn{2}{|c|}{$-132.73 \pm 0.07$} & $-128.70 \pm 0.14$ & $-147.72 \pm 0.09$ & $-139.68 \pm 0.10$ \\
\hline $13-14$ & \multicolumn{2}{|c|}{$-131.44 \pm 0.07$} & $-127.82 \pm 0.14$ & $-145.05 \pm 0.09$ & $-137.62 \pm 0.10$ \\
\hline
\end{tabular}

${ }^{\mathrm{a}} J_{\mathrm{HH}}$ values (from Ref. 18) and $J_{\mathrm{CH}}$ values (from Ref. 19) were kept fixed in the analysis.

$$
C_{\alpha \beta}^{i j}=\sum_{\nu=1}^{3 N}\left(u_{i \alpha}^{(\nu)}-u_{j \alpha}^{(\nu)}\right)\left(u_{i \beta}^{(\nu)}-u_{j \beta}^{(\nu)}\right) \frac{A}{\omega_{\nu}} \operatorname{coth}\left(\frac{B \omega_{\nu}}{T}\right),
$$

$T$ being the temperature, and with $A=h / 8 \pi^{2} c$ and $B$ $=c h / 2 k_{B}$. The calculation of $D_{i j}^{R / E}$ (vib) requires knowledge of $u_{i \alpha}^{(\nu)}$, the Cartesian displacements of the $i$ th nucleus in the $\nu$ th normal mode, and $\omega_{\nu}$, the vibrational frequencies. However, although there are often published values of the $\omega_{\nu}$ and their assignments to normal modes, it is unusual to also have reported values for the $u_{i \alpha}^{(\nu)}$, and this is the case for styrene. It has been shown ${ }^{21}$ for benzene, for which there are values of the frequencies and experimentally derived displacements, that it is possible to calculate acceptable values of $u_{i \alpha}^{(\nu)}$ by either the density functional B3LYP/6-31G* or the MP2/6-31G* methods in the GAUSSIAN 98 suite of programs. In both cases, using calculated values of $u_{i \alpha}^{(\nu)}$ but experimental frequencies $\omega_{\nu}$ for benzene gave values of $D_{i j}(\mathrm{vib})$ in close agreement with those calculated with experimentally derived values of $u_{i \alpha}^{(\nu)}$ and $\omega_{\nu}$. This method of calculating
$D_{i j}$ (vib) using theoretical values of $u_{i \alpha}^{(\nu)}$ together with experimental frequencies is used here for styrene. The frequencies were taken from the analysis by Condirston and Laposa. $^{22}$

There is, however, a general problem in calculating the displacements for a molecule like styrene, which has bond rotational motion. The displacements can be calculated only for the molecule at an energy minimum, but the position of such minima depends on the nature of the calculation. Thus, for styrene, the density functional method B3LYP/6-31G* finds the minimum at $\phi=0^{\circ}$, while the method MP2/6-31G* has minima symmetrically displaced from $0^{\circ}$ and $180^{\circ}$ by $27^{\circ}$. It is necessary, therefore, when exploring conformational models of styrene which are consistent with the dipolar couplings to use B3LYP/6-31G* to calculate the values of $u_{i \alpha}^{(\nu)}$ when assuming that the molecule has a planar minimum energy structure, but to use MP2/6-31G* when this structure is nonplanar.

When averaging the dipolar couplings over a bond rota- 


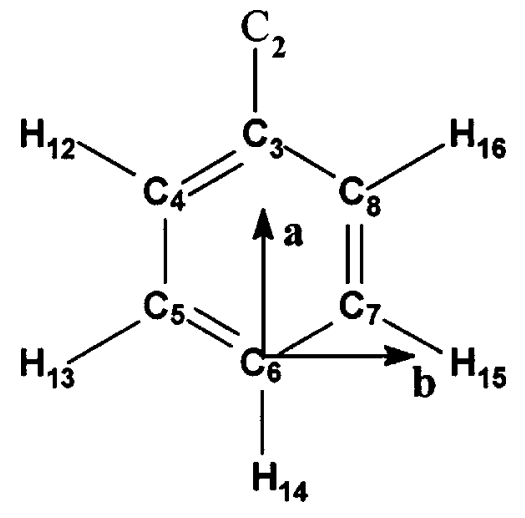

FIG. 3. Location of axes $a b c$ in the phenyl ring. The axis $c$ is normal to the average ring plane.

tion it is important not to average also over a torsional oscillation about the same bond. For this reason the lowestfrequency mode for styrene, which is mainly a torsional oscillation, is not included in the calculation of $D_{i j}(v i b)$.

\section{B. Geometry of the phenyl ring}

A totally unconstrained geometry optimization for styrene by MP2/6-31G* at the energy minimum, which has $\phi$ $=27^{\circ}$, has the ring protons slightly out of plane. The NMR data, however, refer to a structure which is averaged over the whole of the range of $\phi$, and for simplicity this is assumed to have a planar ring. Note too that the averaging over $\phi$ creates a local symmetry of $C_{2 v}$, which is consistent with the observed nuclear spin symmetry for the phenyl protons. With this symmetry and axes $a b c$ located as shown in Fig. 3, the terms in Eq. (1) involving off-diagonal elements of $\mathbf{S}^{R}$ are all zero.

Each isotopomer produces six values of $D_{i j}^{R}$ (obs) from the spectrum in a particular solvent and the unknowns in Eq. (1) are $S_{a a}^{R}, S_{b b}^{R}-S_{c c}^{R}$ and four proton coordinates $a_{12}, b_{12}$, $a_{13}$, and $b_{13}$ [note, however, that a fixed interproton distance is required because only relative internuclear distances can be obtained from Eq. (1)]. Assuming that the ring structure of styrene is solvent independent, as found for benzene, ${ }^{23}$ the values of proton coordinates can be obtained from a simultaneous fit to the values of the intraring $D_{i j}$ of $\mathrm{C} 1$ and $\mathrm{C} 2$ isotopomers of styrene in I35 and ZLI 1132. The use of the data from both the liquid crystals provides extra independent equations to the fitting procedure, making more reliable the structure obtained. $^{24}$ So there are $4 \times 6=24$ values of $D_{i j}^{R}$ (obs), against 12 unknowns that are $\left(S_{a a}^{R}, S_{b b}^{R}\right.$ $\left.-S_{c c}^{R}\right)_{\text {in ZLI }}$ and $\left(S_{a a}^{R}, S_{b b}^{R}-S_{c c}^{R}\right)_{\text {in I35 }}$ for each isotopomer and just 4 common unknown coordinates. The fixed distance was taken to be $r_{13,15}=4.3032 \AA$, the value calculated by MP2/6-31G* when the geometry optimization was carried out with the ring constrained to be planar. The optimization process actually used internal coordinates rather than Cartesians, and these were the bond angle $\mathrm{C}_{3} \mathrm{C}_{4} \mathrm{H}_{12}$ and the bond lengths $r_{4,12}$ and $r_{6,14}$. Preliminary calculations suggested that the dipolar couplings are consistent with the whole molecule being nonplanar, so that the values of $D_{i j}^{R}(\mathrm{vib})$ were calculated using values of $u_{i \alpha}^{(\nu)}$ obtained by MP2/6-31G*.
The results of fitting values of $D_{i j}^{R}$ (equil) calculated with Eq. (1) to observed dipolar couplings so as to minimize the error function $R$,

$$
R=\sqrt{\frac{\sum_{1}^{N}\left(\Delta D_{i j}\right)^{2}}{N}}
$$

where $\Delta D_{i j}=D_{i j}^{R}$ (equil) $+D_{i j}^{R}($ vib $)-D_{i j}^{R}$ (obs), and $N$ is the number of couplings, are given in Table II, where the positions of the ring carbon nuclei calculated by MP2/6-31G* are also given. The values of $D_{i j}^{R}$ (obs) were also fitted to those calculated - that is, neglecting vibrational averagingwhich reveals how vibrational averaging changes the derived geometry.

Note that the optimized values of $r_{4,12}$ are both considerably shorter than the value calculated of $1.088 \AA$, which certainly means that the average distance $r_{12,16}$ is shorter than the calculated value for the molecule in the fixed-energy minimum conformation. It does not mean that the $\mathrm{C}-\mathrm{H}$ bond lengths are necessarily different since the same interproton distance could be obtained by changes in all the bond lengths and angles in the fragment involving $\mathrm{C} 3, \mathrm{C} 4, \mathrm{C} 8$, and $\mathrm{H} 12$ and $\mathrm{H} 16$.

It is interesting to note that the values of $D_{i j}^{R}$ (vib) are all $<1 \%$ of $D_{i j}^{R}$ (equil), but it is difficult to estimate the precision of these values and, hence, of the derived geometrical parameters for the ring. As noted earlier, there is no simple way that this can be done. If the molecule is assumed to be planar, the values of $D_{i j}^{R}$ (vib) should be obtained using the density functional method B3LYP/6-31G*. Doing this leads to changes in the values of $D_{i j}^{R}$ (vib) shown in Table II, which also shows the results of using these to obtain the geometrical parameters. The differences in the bond lengths and angles are small, and clearly for the phenyl ring whether MP2/6-31G* or B3LYP/6-31G* is used to calculate $D_{i j}^{R}(\mathrm{vib})$ is unimportant, which is to be expected when the vibrational corrections are small. The small magnitude $D_{i j}^{R}$ (vib) for interproton couplings also means that the geometry obtained when vibrational averaging is neglected is very similar to when it is included.

The optimized positions of the ring nuclei were then kept fixed in all subsequent calculations of dipolar couplings.

\section{Structure of the ene fragment}

To calculate the geometries of the ene fragment, we started with the same approach followed for the ring (note that the calculation of the displacements proceeds using the axes $x y z$ fixed in the ene fragment, and not the axes $a b c$ fixed in the phenyl ring). The local symmetry of the ene group, if this fragment is planar, is $C_{s}$, and so with the axes fixed as shown in Fig. 1 there is just one nonzero off-diagonal element $S_{x z}^{E}$. Each isotopomer produces 6 values of $D_{i j}^{E}(\mathrm{obs})$ from the spectrum in a particular solvent. There are 7 coordinates defining the relative positions of the interacting nuclei, and 3 order parameters are required to calculate values of $D_{i j}^{E}$ (equil). The number of unknowns is reduced to 9 when one internuclear distance is fixed at $1.343 \AA$, and there is therefore insufficient data from the spectrum of a single isotopomer in one solvent from which to obtain optimized 
TABLE II. Vibrational contribution to dipolar couplings $D_{i j}^{R}$ (vib), $\Delta D_{i j}=D_{i j}(\mathrm{obs})-D_{i j}$ (calc), and the bond lengths and angles obtained for the phenyl ring using the MP2/6-31G* or B3LYP/6-31G* level of approximation to calculate the vibrational force field. The geometry obtained by neglecting the effect of vibrational averaging is also included.

\begin{tabular}{|c|c|c|c|c|c|}
\hline & & Force & MP2 & Force & BLYP \\
\hline & $i, j$ & $D_{i j}^{R}(\mathrm{vib})$ & $\Delta D_{i j}$ & $D_{i j}^{R}(\mathrm{vib})$ & $\Delta D_{i j}$ \\
\hline & 12,13 & 27.83 & -0.04 & 22.17 & -0.02 \\
\hline & 12,14 & 3.68 & 0.51 & 2.00 & 0.37 \\
\hline ZLI 1132 & 12,15 & 0.37 & 0.14 & 0.23 & 0.62 \\
\hline (C2) & 12,16 & 0.25 & 0.27 & 0.24 & 0.29 \\
\hline & 13,14 & 12.22 & 0.17 & 6.62 & -0.21 \\
\hline & 13,15 & 0.18 & 0.61 & 0.13 & 0.51 \\
\hline & 12,13 & 27.14 & -0.11 & 21.62 & -0.09 \\
\hline & 12,14 & 3.59 & 0.84 & 1.95 & 0.70 \\
\hline ZLI 1132 & 12,15 & 0.36 & 0.18 & 0.22 & 0.65 \\
\hline (C1) & 12,16 & 0.24 & 0.31 & 0.23 & 0.32 \\
\hline & 13,14 & 11.90 & 0.08 & 6.45 & -0.12 \\
\hline & 13,15 & 0.17 & 0.28 & 0.13 & -0.38 \\
\hline & 12,13 & 28.29 & 0.03 & 22.63 & 0.04 \\
\hline & 12,14 & 3.71 & -0.39 & 2.02 & -0.50 \\
\hline $\mathrm{I} 35$ & 12,15 & 0.25 & -0.56 & 0.13 & -0.04 \\
\hline (C2) & 12,16 & -0.07 & 0.27 & -0.01 & 0.34 \\
\hline & 13,14 & 11.04 & 0.17 & 5.88 & 0.13 \\
\hline & 13,15 & -0.14 & -0.81 & -0.11 & -1.05 \\
\hline & 12,13 & 26.85 & 0.02 & 21.48 & 0.02 \\
\hline & 12,14 & 3.52 & -0.39 & 1.92 & -0.50 \\
\hline I35 & 12,15 & 0.24 & -0.63 & 0.13 & -0.14 \\
\hline (C1) & 12,16 & -0.06 & 0.45 & -0.01 & 0.51 \\
\hline & 13,14 & 10.49 & 0.24 & 5.59 & 0.21 \\
\hline & 13,15 & -0.13 & -1.52 & -0.11 & -1.75 \\
\hline
\end{tabular}

\begin{tabular}{lcccr} 
& \multicolumn{2}{c}{$\begin{array}{c}\text { Optimized geometries } \\
\text { B3LYP }\end{array}$} & $\begin{array}{c}\text { MP2 } \\
\text { None }^{\mathrm{c}}\end{array}$ & $\begin{array}{c}\text { MP2/6-31G } \\
\text { optimized }^{*}\end{array}$ \\
\hline$r_{4,12}(\AA)$ & $1.060 \pm 0.004$ & $1.065 \pm 0.04$ & $1.057 \pm 0.05$ & 1.088 \\
$r_{5,13}(\AA)$ & $1.088^{\mathrm{e}}$ & $1.088^{\mathrm{e}}$ & $1.088^{\mathrm{e}}$ & 1.088 \\
$r_{6,14}(\AA)$ & $1.092 \pm 0.002$ & $1.093 \pm 0.002$ & $1.090 \pm 0.002$ & 1.087 \\
$<_{12,4,3}(\mathrm{deg})$ & $119.8 \pm 0.03$ & $119.6 \pm 0.03$ & $119.7 \pm 0.03$ & 119.6 \\
$<_{13,5,4}(\mathrm{deg})$ & $119.7^{\mathrm{e}}$ & $119.7^{\mathrm{e}}$ & $119.7^{\mathrm{e}}$ & 119.7 \\
$<_{14,6,5}(\mathrm{deg})$ & $120.2^{\mathrm{e}}$ & $120.2^{\mathrm{e}}$ & $120.2^{\mathrm{e}}$ & 120.2 \\
\hline
\end{tabular}

${ }^{a}$ Force field calculated by B3LYP/6-31G* with the whole molecule planar.

${ }^{\mathrm{b}}$ Force field calculated with MP2/6-31G* with ene group rotated $27^{\circ}$ out of phenyl ring plane.

${ }^{\mathrm{c}}$ Without vibrational averaging.

${ }^{\mathrm{d}}$ Geometry calculated.

eParameter kept fixed.

values of the 9 unknown parameters. Using (C1) and (C2) intra-ene experimental couplings from both the mesophases and assuming (as previously done for the ring) the ene structure to be solvent independent made the problem overdetermined, having $24 D_{i j}^{E}$ (obs) vs 18 unknowns $(3 \times 4=12$ order parameters +6 common geometrical parameters). However, this gave a large rms and too short $\mathrm{CH}$ distances $(<1.04 \AA$ ). At this point, we restricted the data sets to just the data obtained for both isotopomers in a single solvent and assumed that the structure is the same for both labeled species. This produces 12 values of $D_{i j}^{E}$ (obs) and the unknowns also become 12: 6 order parameters ( 3 for each isotopomer) and 6 geometrical parameters. The values of $D_{i j}^{E}$ (vib) were calculated by the MP2/6-31G* method, which is appropriate if the whole molecule is nonplanar. Carrying out this procedure for data for the two solvents yielded exact fits to the data (12 pieces of data, 12 variables), but with unacceptable results for the geometries: thus, the $\mathrm{C}-\mathrm{H}$ bond lengths are all found to be $<0.7 \AA$.

One possible reason for the failure to obtain a reasonable optimized geometry may be that the two isotopomers have order parameters which are very close in value and almost linearly related to one another, so that the two data sets are not linearly independent. To remove this problem an average set of 9 values of $D_{i j}^{E}(\mathrm{obs})$ was constructed by taking the data for the $(\mathrm{C} 1)$ isotopomer and adding the three ${ }^{13} \mathrm{C}-{ }^{1} \mathrm{H}$ couplings obtained for the $(\mathrm{C} 2)$ isotopomer in the same solvent and scaled by the average ratio of the three observed couplings between the ene protons [this procedure gives 9 values of $D_{i j}^{E}$ (obs) compared with 3 order parameters and so again it is possible optimize 6 geometrical parameters]. A perfect fit is obtained for each set of data for the two sol- 
TABLE III. Comparison of the values of $D_{i j}^{E}(\mathrm{vib}) / D_{i j}^{E}$ (obs) calculated for a planar ene fragment, but with either the MP2/6-31G* (with whole molecule nonplanar) B3LYP/6-31G* (with whole molecule planar) force fields.

\begin{tabular}{crcrrr}
\hline \hline \multicolumn{4}{c}{$D_{i j}^{E}(\mathrm{vib}) / D_{i j}^{E}(\mathrm{obs})(\%)$} \\
\cline { 5 - 6 }$i, j$ & \multicolumn{2}{c}{$\begin{array}{c}\text { ZLI 1132 } \\
\text { combined data }\end{array}$} & \multicolumn{2}{c}{$\begin{array}{c}\text { I35 } \\
\text { combined data }\end{array}$} \\
\cline { 2 - 3 } \cline { 5 - 6 } \cline { 5 - 6 } 1,9 & MP2 & B3LYP & & MP2 & B3LYP \\
1,10 & -10.9 & -9.3 & & -10.8 & -9.3 \\
1,11 & -2.1 & -8.0 & & -6.2 & -7.7 \\
2,9 & -45.0 & -1.3 & & 6.3 & 0.4 \\
2,10 & -2.2 & -2.2 & & -2.1 & -2.2 \\
2,11 & -21.5 & -3.8 & & 12.5 & 2.1 \\
9,10 & 6.9 & 3.3 & & -2.3 & -4.7 \\
9,11 & -11.8 & -5.2 & & -12.1 & -5.1 \\
10,11 & -0.3 & 0.1 & & -0.3 & 0.2 \\
\hline \hline
\end{tabular}

vents, but again with an unacceptably distorted geometry.

The geometry was then restricted to being closer to that expected from the molecular orbital (MO) calculations: thus, the value of $r_{19}$ was kept fixed at $1.083 \AA$ and $r_{12}$ $=1.343 \AA$, which agrees with the MP2 calculation. The optimized geometry is now within acceptable limits, but there are very large values of $\Delta D_{1,11}=7.0$ (ZLI 1132) and 11.7 (I35) $\mathrm{Hz}$ and $\Delta D_{2,10}=-6.7$ (ZLI 1132) and -7.6 (I35) $\mathrm{Hz}$. It appears, also by looking at the values of $\Delta D_{1,11}$ and $\Delta D_{2,10}$ for each solvent (about equal in magnitude but opposite in sign), that the combined data set for the $\mathrm{C} 1$ and $\mathrm{C} 2$ isotopomers for each solvent shows that a planar ene geometry is incompatible with the data. Two possible reasons for the failure to obtain a reasonable planar geometry so far for the ene fragment have been explored. Thus the values of $D_{i j}^{E}$ (vib) may be inaccurate, and the ene fragment may not be exactly planar.

The calculated values of $D_{i j}^{E}(\mathrm{vib}) / D_{i j}^{E}(\mathrm{obs})$, with $r_{19}$ fixed at $1.083 \AA$, are in fact very large, and to have some estimate of how accurate these values are, which were calculated with the MP2/6-31G* method with the whole molecule nonplanar, but planar ene and ring fragments, they were recalculated with the B3LYP/6-31G* method, with the whole molecule planar. The results are shown in Table III.

The largest values of $D_{i j}^{E}(\mathrm{vib}) / D_{i j}^{E}(\mathrm{obs})$ are obtained for the smallest couplings $(1,11$ in ZLI 1132, and 2,10 in ZLI 1132 ), and these also show the largest differences when different force fields are used. This is because the internuclear vectors for these pairs of nuclei for the sample dissolved in ZLI 1132 are, on average, oriented with respect to the liquid crystal director at angles close to $54.7^{\circ}$, where the sensitivity of the dipolar couplings to angular fluctuations is the greatest.

The method for calculating the force field is clearly important for determining the values of $D_{i j}^{E}$ (vib), but the dilemma remains that the two methods used here, which both give good results when applied to calculating vibrational corrections of dipolar couplings for benzene, where they both give the same symmetry for the minimum energy structure, produce different optimized structures and values of $D_{i j}^{E}(\mathrm{vib})$ for styrene. We will, therefore, continue to use both force
TABLE IV. Geometrical variables used in the four-step process of obtaining best agreement between observed and calculated dipolar couplings for the ene fragment of styrene.

\begin{tabular}{cc}
\hline \hline Step No. & Varied parameters \\
\hline 1 & $r_{1,9} ; r_{1,10} ; r_{2,11}$ \\
2 & $<_{9,1,2} ;<_{10,1,2} ;<_{11,2,1}$ \\
3 & $<_{10,1,2} ;<_{9,1,2,3} ;<_{11,2,1,10}$ \\
4 & $r_{2,11} ;<_{11,2,1} ;<_{10,1,2,3}$ \\
\hline \hline
\end{tabular}

fields to calculate vibrational corrections in order to judge how important this is in determining the structure and conformation of styrene from dipolar couplings.

To explore the possibility that the ene fragment is not planar, it is noted that a molecular orbital calculation with full geometry optimization with MP2/6-31G* produces a small deviation from planarity of the ene fragment. Starting with this geometry and using the combined data sets for the two solvents (nine couplings in each case), a nonplanar geometry was obtained which gives the best fit to the dipolar couplings. The optimization process proceeded in four steps. In each step the variables were five order parameters and three geometrical parameters, as shown in Table IV.

The calculations were repeated, but using the force field calculated by B3LYP with the calculated planar geometry, and both sets of results are shown in Table V, where the values of $\Delta D_{i j}^{E}$ are given for the final step. The two methods of calculating $D_{i j}^{E}$ (vib) give very different values for many of the dipolar couplings, and it is not possible to decide which is the better set of values: the calculations with MP2 are consistent for styrene in obtaining the force field with a nonplanar geometry, but B3LYP, at least for benzene, is thought to produce the most accurate force fields. The bond lengths obtained by the two calculations differ significantly, but the angles are very close, as shown in Table V.

The calculations on a nonplanar ene fragment were repeated, but with the neglect of vibrational averaging, and the results are also given in Table $\mathrm{V}$.

Both calculations involving vibrational corrections agree on a nonplanar structure and in subsequent calculations the structure determined using the MP2 force field is used. The neglect of vibrational averaging gives a smaller deviation from the $\mathrm{C}_{1} \mathrm{C}_{2} \mathrm{C}_{3}$ plane $\left(-1.0^{\circ}\right.$ against $+2.35^{\circ}$ for MP2 and $+2.0^{\circ}$ for B3LYP), but also produced $\mathrm{C}-\mathrm{H}$ bond lengths which are 3\%-4\% longer than those predicted by MO calculations and a higher rms of $2.8 \mathrm{~Hz}$. This is consistent with previous observations that neglecting vibrational averaging of dipolar couplings leads to the determination of longer $\mathrm{C}-\mathrm{H}$ bond lengths. ${ }^{9,25}$

Note that it has been assumed that the fragment structures do not change as they rotate about the ring-ene bond. The calculations by Cochrane $\mathrm{et} \mathrm{al.}^{3}$ find that there are small changes as the angle $\phi$ changes, but these are confined to the region near $\phi=0^{\circ}$, and the same calculations find that the molecule spends most time with $\phi=27^{\circ}$. Consequently, it is reasonable to neglect such geometrical distortions in the con- 
TABLE V. Vibrational contribution to dipolar couplings $D_{i j}$ (vib), $\Delta D_{i j}=D_{i j}(\mathrm{obs})-D_{i j}(\mathrm{calc})$, and the geometrical parameters obtained by fitting observed to calculated dipolar couplings when the ene fragment is allowed to be nonplanar. The vibrational calculations were obtained with either the force field calculated by the MP2/6-31G* or B3LYP/6-31G* methods. The geometrical parameters obtained when vibrational averaging is neglected are also shown.

\begin{tabular}{|c|c|c|c|c|c|c|}
\hline \multicolumn{2}{|l|}{ Force field } & \multicolumn{2}{|c|}{ MP2 } & \multicolumn{2}{|c|}{ B3LYP } & \multirow{2}{*}{$\begin{array}{r}\text { None } \\
1.54\end{array}$} \\
\hline \multirow{9}{*}{$\begin{array}{l}\text { ZLI 1132: } \\
\text { combined } \\
\text { data from } \\
\text { both } \\
\text { isotopomers }\end{array}$} & 1,9 & 704.88 & -0.32 & 597.85 & -0.15 & \\
\hline & 1,10 & -16.53 & -0.04 & -70.74 & 0.13 & 2.27 \\
\hline & 1,11 & 5.79 & -1.95 & 0.27 & -2.12 & 1.86 \\
\hline & 2,9 & 20.54 & 1.18 & 20.96 & 0.65 & -1.44 \\
\hline & 2,10 & 6.11 & -0.15 & 0.86 & -0.79 & -1.19 \\
\hline & 2,11 & 46.28 & 0.10 & -19.46 & 0.11 & -3.07 \\
\hline & 9,10 & 232.31 & 0.25 & 103.58 & -0.03 & 0.04 \\
\hline & 9,11 & 4.16 & 0.40 & -0.94 & 0.52 & 0.38 \\
\hline & 10,11 & 2.96 & 0.27 & -3.86 & 0.38 & 2.80 \\
\hline & 1,9 & 734.22 & 0.31 & 625.30 & 0.16 & 0.88 \\
\hline & 1,10 & -104.20 & -0.04 & -128.99 & -0.23 & 0.22 \\
\hline I35: & 1,11 & 4.04 & 0.96 & -0.11 & 0.79 & 6.73 \\
\hline combined & 2,9 & 22.00 & -1.20 & 22.42 & -0.78 & -4.49 \\
\hline data from & 2,10 & 4.74 & -0.05 & 0.55 & 0.42 & -5.36 \\
\hline both & 2,11 & -33.82 & -0.11 & -65.94 & -0.13 & -0.64 \\
\hline \multirow[t]{3}{*}{ isotopomers } & 9,10 & 203.66 & -0.20 & 87.60 & 0.06 & 0.98 \\
\hline & 9,11 & 3.02 & -0.30 & -2.19 & -0.38 & 4.01 \\
\hline & 10,11 & 1.50 & 0.89 & -5.29 & 1.28 & 0.27 \\
\hline Parameters & & Values & & Values & & Values \\
\hline$r_{1,2}$ & & 1.343 & & 1.343 & & 1.343 \\
\hline$r_{9,1}$ & & $1.083 \pm 0.001$ & & $1.088 \pm 0.001$ & & $1.130 \pm 0.003$ \\
\hline$r_{10,1}$ & & $1.084 \pm 0.001$ & & $1.094 \pm 0.001$ & & $1.120 \pm 0.001$ \\
\hline$r_{11,2}$ & & $1.080 \pm 0.006$ & & $1.090 \pm 0.005$ & & $1.113 \pm 0.003$ \\
\hline$<_{9,1,2}$ & & $121.06 \pm 0.10$ & & $121.1 \pm 0.1$ & & $121.2 \pm 0.2$ \\
\hline$<_{10,1,2}$ & & $122.6 \pm 0.1$ & & $122.7 \pm 0.1$ & & $122.4 \pm 0.1$ \\
\hline$<_{11,2,1}$ & & $118.8 \pm 0.1$ & & $118.8 \pm 0.1$ & & $118.8 \pm 0.1$ \\
\hline$<_{9,1,2,3}$ & & $181.4 \pm 0.5$ & & $181.7 \pm 0.5$ & & $179.9 \pm 0.4$ \\
\hline$<_{10,1,2,3}$ & & $1.1 \pm 0.5$ & & $1.2 \pm 0.4$ & & $0.9 \pm 7.6$ \\
\hline$<_{11,2,1,10}$ & & $183.4 \pm 1.4$ & & $183.7 \pm 1.1$ & & $178.9 \pm 1.7$ \\
\hline
\end{tabular}

formational analysis, which also finds a high probability that the molecule is not in a sterically hindered structure.

\section{Conformational analysis}

The dipolar couplings in a molecule undergoing largeamplitude motion, such as rotation about the ring-ene bond, which is fast compared with the changes produced in the couplings, are averaged according to

$$
D_{i j}(\text { equil })=\int D_{i j}(\text { equil }, \phi) P_{L C}(\phi) d \phi,
$$

where $P_{L C}(\phi)$ is the probability that the angle $\phi$ is between $\phi$ and $\phi+d \phi$. The values of $D_{i j}$ (equil, $\phi$ ) are given by

$$
\begin{aligned}
D_{i j}(\text { equil }, \phi)= & -\left(K_{i j} / r_{i j}^{3}\right)\left\{S_{a a}(\phi)\left(3 \cos ^{2} \theta_{i j a}-1\right)\right. \\
& +\left[S_{b b}(\phi)-S_{c c}(\phi)\right]\left(\cos ^{2} \theta_{i j b}-\cos ^{2} \theta_{i j c}\right) \\
& +4 S_{a b}(\phi) \cos \theta_{i j a} \cos \theta_{i j b}+4 S_{a c}(\phi) \\
& \times \cos \theta_{i j a} \cos \theta_{i j c}+4 s_{b c}(\phi) \\
& \left.\times \cos \theta_{i j b} \cos \theta_{i j c}\right\} .
\end{aligned}
$$

The distances $r_{i j}$ and angles $\theta_{i j \alpha}$ are in general dependent on $\phi$.
In order to calculate the conformationally dependent order parameters $S_{a b}(\phi)$, it is necessary to adopt a theoretical model of how the solute-solvent, anisotropic potential $U_{L C}(\beta, \gamma, \phi)$ depends on the orientation of the liquid crystal director in a molecule-fixed frame, specified by polar angles $\beta$ and $\gamma$, when in the conformation specified by $\phi$. The additive potential (AP) method is adopted here, ${ }^{26}$ which expresses $U_{L C}(\beta, \gamma, \phi)$ as

$$
U_{L C}(\beta, \gamma, \phi)=U_{\text {ext }}(\beta, \gamma, \phi)+U_{\text {iso }}(\phi),
$$

where $U_{\text {ext }}(\beta, \gamma, \phi)$ is a purely anisotropic potential of mean torque and $U_{\text {iso }}(\phi)$ is nonzero in both the liquid crystalline and isotropic phases. The order parameters are given by

$$
\begin{aligned}
S_{a b}(\phi)= & Z(\phi)^{-1} \int\left(\frac{3 \cos \theta_{a} \cos \theta_{b}-\delta_{a b}}{2}\right) \\
& \times \exp \left[-\frac{U_{\text {ext }}(\beta, \gamma, \phi)}{k_{B} T}\right] \sin \beta d \beta d \gamma,
\end{aligned}
$$

with

$$
Z(\phi)=\int \exp \left[-\frac{U_{\text {ext }}(\beta, \gamma, \phi)}{k_{B} T}\right] \sin \beta d \beta d \gamma
$$

and $\delta_{a b}=1$ if $a=b$ and zero otherwise. 
In the AP method $U_{\text {ext }}(\beta, \gamma, \phi)$ is approximated as

$$
\begin{aligned}
U_{\text {ext }}(\beta, \gamma, \phi)= & -\varepsilon_{2,0}(\phi) C_{2,0}(\beta) \\
& -2 \operatorname{Re} \varepsilon_{2,2}(\phi) C_{2,2}(\beta, \gamma),
\end{aligned}
$$

where the $\varepsilon_{2, m}(\phi)$ are conformationally dependent, solutesolvent interaction parameters, and the $C_{2, m}(\beta, \gamma)$ are modified spherical harmonics. The distinguishing feature of the AP method is that the $\varepsilon_{2, m}(\phi)$ are related to conformationally independent interaction parameters $\varepsilon_{2, m}(j)$ for each rigid fragment $j$ in the molecule by

$$
\varepsilon_{2, m}(\phi)=\sum_{j} \sum_{p} \varepsilon_{2, p}(j) D_{p, m}^{2}\left(\Omega_{j}\right) .
$$

The Wigner function $D_{p, m}^{2}\left(\Omega_{j}\right)$ describes the orientation of the fragment $j$ in a molecule-fixed reference frame. For styrene there are two rigid fragments: the ring and the ene group. The ring is assumed to have $C_{2 v}$ symmetry and which therefore requires $\varepsilon_{2,0}(R)$ and $\varepsilon_{2,2}(R)$. The nonplanar ene group was represented by axially symmetric interaction tensors $\varepsilon_{2,0}(\mathrm{CH})$ pointing along each of the $\mathrm{C}-\mathrm{H}$ bonds and one, $\varepsilon_{2,0}(\mathrm{CC})$, along the $\mathrm{C}=\mathrm{C}$ bond. In practice, it is more convenient to work with Cartesian tensor components, which are related to the values of the $\varepsilon_{2, m}$ by

$$
\begin{aligned}
& \varepsilon_{a a}(R)=(2 / 3)^{1 / 2} \varepsilon_{2,0}(R), \\
& \varepsilon_{b b}(R)-\varepsilon_{c c}(R)=2 \varepsilon_{2,2}(R), \\
& \varepsilon_{C H}=(2 / 3)^{1 / 2} \varepsilon_{2,0}(\mathrm{CH}), \\
& \varepsilon_{C C}=(2 / 3)^{1 / 2} \varepsilon_{2,0}(\mathrm{CC}) .
\end{aligned}
$$

Note that the $\mathrm{C} 2-\mathrm{C} 3$ bond is part of the ene fragment, but is also colinear with the ring $a$ axis since the ring has been assumed to have $C_{2 v}$ symmetry. This means that with the above four interaction parameters the correct symmetries are predicted for the local order matrices, and in particular there are five nonzero, independent values of $S_{\alpha \beta}^{E}(\alpha, \beta$ being $x, y$, $z$ in turn).

To calculate values of $D_{i j}$ (equil) it is necessary to adopt a model for $P_{L C}(\phi)$. This is related to $P_{L C}(\beta, \gamma, \phi)$, the probability that the molecule is in a conformation defined by $\phi$, while being at an orientation with respect to the liquid crystal director, described by $\beta$ and $\gamma$. Thus,

$$
P_{L C}(\phi)=\int P_{L C}(\beta, \gamma, \phi) \sin \beta d \beta d \gamma
$$

and

$$
P_{L C}(\beta, \gamma, \phi)=Q^{-1} \exp \left[-U_{L C}(\beta, \gamma, \phi) / k_{B} T\right],
$$

with

$$
Q=\int \exp \left[-U_{L C}(\beta, \gamma, \phi) / k_{B} T\right] \sin \beta d \beta d \gamma d \phi .
$$

Note that $P_{L C}(\phi)$ is not equal to $P_{\text {iso }}(\phi)$, the probability distribution in an isotropic phase of the liquid crystalline solvent, which is given by

$$
P_{\text {iso }}(\phi)=Q_{\text {iso }}^{-1} \exp \left[-U_{\text {int }}(\phi) / k_{B} T\right],
$$

with
TABLE VI. Bond length $r_{2,3}$, the angles $\alpha$ and $\beta$ (see Fig. 1), and the position $\phi_{0}$ and width $h$ of the Gaussian functions obtained by fitting calculated to observed dipolar couplings obtained for samples of ${ }^{13} \mathrm{C} 1$ - styrene and ${ }^{13} \mathrm{C} 2$-styrene dissolved in the nematic solvents ZLI 1132 and I35 compared with values calculated by MP2/6-31G*. Vibrational averaging was done using force field calculated by the MP2/6-31G

\begin{tabular}{ccc}
\hline \hline Parameters & $\begin{array}{c}\text { This } \\
\text { work }\end{array}$ & $\begin{array}{c}\text { Calculation by } \\
\text { MP2/6-31G* }\end{array}$ \\
\hline$r_{2,3}(\AA)$ & $1.464 \pm 0.004$ & 1.4719 \\
$\alpha(\mathrm{deg})$ & $124.03 \pm 0.08$ & 122.06 \\
$\beta(\mathrm{deg})$ & $124.43 \pm 0.07$ & 125.26 \\
$\mathrm{~h}(\mathrm{deg})$ & $11.2 \pm 1.5$ & - \\
$($ in ZLI 1132) & & 27 \\
$\phi_{0}(\mathrm{deg})$ & $18.2 \pm 0.3$ & - \\
(in ZLI 1132) & & \\
$\mathrm{h} \mathrm{(deg)}$ & $13.0 \pm 1.6$ & - \\
(in I35) & & \\
$\phi_{0}(\mathrm{deg})$ & $17.9 \pm 0.5$ & \\
(in I35) & & \\
rms (Hz) & 2.07 & \\
\hline \hline
\end{tabular}

$$
Q_{\text {iso }}=\int \exp \left[-U_{\text {iso }}(\phi) / k_{B} T\right] d \phi .
$$

The remaining step to calculate the values of $D_{i j}$ (equil) is to adopt a model for $U_{\text {int }}(\phi)$, which together with $U_{\text {ext }}(\beta, \gamma, \phi)$ determines the form of $P_{L C}(\phi)$. The usual approach when averaging over a bond rotation is to adopt a functional form for $V(\phi)$. Cochrane et al. ${ }^{3}$ used a function

$$
V(\phi)=V_{0}\left\{1-2\left(\phi / \phi_{0}\right)^{2}+\left(\phi / \phi_{0}\right)^{4}\right\},
$$

with $\phi_{0}=27^{\circ}$ and $V_{0}=0.243 \mathrm{kcal} \mathrm{mol}^{-1}$, but this does not fit the whole of the curve. Fitting the same data ${ }^{3}$ to a Fourier series produces close agreement with all calculated points with the function

$$
V(\phi)=V_{0}+\sum_{n=1}^{5} V_{n} \cos n \phi,
$$

with $\quad V_{0}=2.467 \mathrm{~kJ} \mathrm{~mol}^{-1}, \quad V_{1}=-3.969 \mathrm{~kJ} \mathrm{~mol}^{-1}, \quad V_{2}$ $=2.383 \mathrm{~kJ} \mathrm{~mol}^{-1}, \quad V_{3}=-1.306 \mathrm{~kJ} \mathrm{~mol}^{-1}, \quad V_{4}$ $=0.528 \mathrm{~kJ} \mathrm{~mol}^{-1}$, and $V_{5}=-0.052 \mathrm{~kJ} \mathrm{~mol}^{-1}$.

It is not admissible, however, to average the dipolar couplings over this surface and use vibrational averaging: the Cartesian displacements are strictly valid only at the energy minimum calculated by the MP2 method. Even if using these displacements over the whole surface were considered to be a reasonable approximation, it is found that attempts to iterate on Fourier coefficients for a potential function of this kind in order to fit calculated to observed couplings does not lead to an acceptable solution, giving very large rms values and severely distorted curves. This is primarily because the main features-positions of turning points and heights of maxima-cannot be changed independently by varying the Fourier coefficients.

A simpler and often more realistic method is to model $P_{L C}(\phi)$ directly. ${ }^{27}$ This approach has been applied here by assuming $P_{L C}(\phi)$ to be a sum of four symmetrically related Gaussian functions of the form 


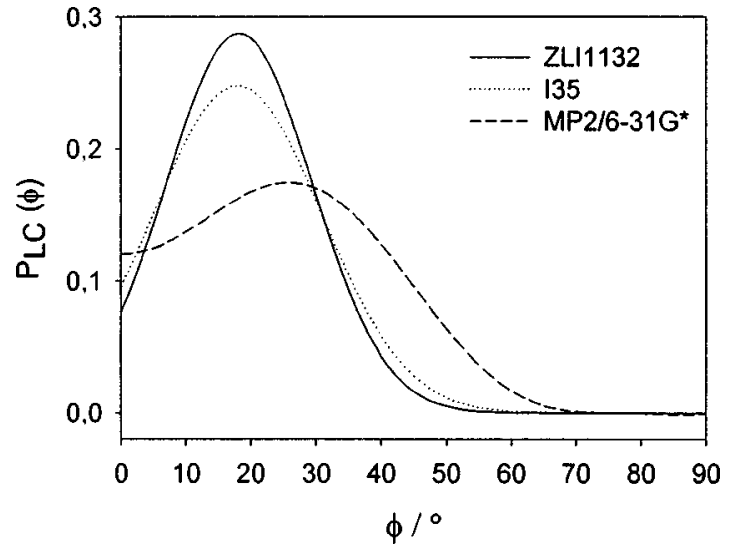

FIG. 4. The probabilities $P_{L C}(\phi)$ normalized over the angular range $0^{\circ}-90^{\circ}$ obtained for styrene dissolved in ZLI 1132 and I35, compared with that calculated by the MP2/6-31G* method (Ref. 3).

$$
P_{L C}(\phi)=\frac{1}{4 h \sqrt{2} \pi} \exp \left[-\frac{\left(\phi-\phi_{0}\right)^{2}}{2 h^{2}}\right],
$$

where $\phi_{0}$ is the center of the function located in the interval $0^{\circ}-90^{\circ}$, with a width at half maximum height of $h$. Now the position and widths of the Gaussian functions are independent variables.

Another advantage of this approach is that vibrational averaging, albeit in an approximate way, can be included if the widths of the Gaussian functions are not large. Thus the force field is calculated for a molecule in a fixed structure with the value of $\phi_{0}$ corresponding to that for the minimum energy form. For styrene the MP2/6-31G* calculation finds $\phi_{0}$ to be $27^{\circ}$. In the calculations by the AP method the location of the Gaussian functions is allowed to vary, but the values of $D_{i j}$ (vib) are still those calculated with $\phi_{0}$ as $27^{\circ}$. The calculations proceeded again by fitting four data sets simultaneously (24 couplings for each isotopomer in each phase), varying $\varepsilon_{a a}(R), \varepsilon_{b b}(R)-\varepsilon_{c c}(R), \varepsilon_{\mathrm{CH}}(R)$, and $\varepsilon_{\mathrm{CC}}$ as phase and isotopomer-dependent variables, $\phi_{0}$ and $h$ as only phase dependent, and the bond distance $r_{23}$ and angles $\alpha$ and $\beta$ as identical in all phases and for each isotopomer. The results are given in Table VI, and Fig. 4 shows the dependence found of $P_{L C}(\phi)$ with $\phi$.

The values of $r_{23}, \alpha$, and $\beta$ are close to those calculated by MP2/6-31G*. The values found for $\phi_{0}$ are essentially the same for the two solvents, but significantly smaller than the value calculated by MP2/6-31G*.

The $P_{L C}(\phi)$ are, in general, affected by the orientational order and will differ from $P_{\text {iso }}(\phi)$, the distribution for the same temperature, but for an isotropic phase. ${ }^{15,26,27}$ In the present case the differences are negligible.

\section{CONCLUSIONS}

The use of two ${ }^{13} \mathrm{C}$-labeled isotopomers of styrene has made it possible to establish experimentally that the ene fragment of styrene is not strictly planar, which is agreement with advanced molecular orbital calculations. To do this it was essential to allow for vibrational averaging of the dipolar couplings.
It has been shown that the probability distribution for the ring-ene bond rotation angle $\phi$, assuming that this has a Gaussian form, has a maximum in the interval $0-90^{\circ}$ at an average of $\phi_{0}=18.0^{\circ} \pm 0.2^{\circ}$, with a width at half height of $h=12.0^{\circ}$. This refers strictly to the molecule in the two solvents which form a liquid crystalline phase, I35 and ZLI 1132, which were used to reveal partially averaged dipolar couplings, but the result is valid for both the liquid crystalline and equivalent isotropic phases. The values of $\phi_{0} \mathrm{ob}-$ tained are significantly different from that calculated of $27^{\circ}$ by the method MP2/6-31G*, which refers to a single, isolated molecule. The difference in the two values can be attributed in part to a difference in the environment of the molecule, that from NMR referring to a liquid environment.

The planarity of styrene, in the sense of the position of the minimum in the bond rotational probability distribution, has excited much attention by experimentalists and theorists. The present experimental study shows that a definitive answer can be obtained using the values of partially averaged dipolar couplings obtained from the proton spectra of samples dissolved in liquid crystalline solvents, but only when ${ }^{13} \mathrm{C}$ labeling is used to establish the structures of the ring and ene fragments. To do this it was necessary to average over the vibrational motions, and it is proposed here that this may be done using vibrational wave functions calculated by molecular orbital methods or density functional methods.

\section{ACKNOWLEDGMENT}

This work has been supported by MURST PRIN ex $40 \%$.

${ }^{1}$ M. Frisch et al., computer code Gaussian 98, Gaussian Inc., Pittsburgh, 1998.

${ }^{2}$ M. Head-Gordon and J. A. Pople, J. Phys. Chem. 97, 1147 (1993).

${ }^{3}$ J. C. Cochrane, K. Hagen, G. Paulen, Quang Shen, S. Tom, M. Traetteberg, and C. Wells, J. Mol. Struct. 413, 313 (1997).

${ }_{4}^{4}$ J. M. Granadino-Roldán, M. Fernández-Gómez, and A. Navarro, Chem. Phys. Lett. 372, 255 (2003).

${ }^{5}$ W. M. Ralowski, P. J. Mjoberg, and S. O. Ljungren, J. Mol. Struct. 30, 1 (1976).

${ }^{6}$ W. M. Ralowski, P. J. Mjoberg, and S. O. Ljungren, J. Mol. Struct. 31, 169 (1976).

${ }^{7}$ NMR of Liquid Crystals, edited by J. W. Emsley (Reidel, Dordrecht, 1985).

${ }^{8}$ J. W. Emsley, in Encylopedia of NMR, edited by D. M. Grant and R. K. Harris (Wiley, Chichester, 1996), pp. 2781, 2788.

${ }^{9}$ P. Diehl, in Encylopedia of NMR (Ref. 8), p. 4591.

${ }^{10}$ J. A. B. Lohman and C. MacLean, Chem. Phys. 35, 269 (1978).

${ }^{11}$ K. L. Facchini, S. W. Stalet, P. C. M. Van Zyk, P. K. Mishra, and A. A. Bothner-By, J. Am. Chem. Soc. 110, 4900 (1988).

${ }^{12}$ A. A. Bothner-By, in Encylopedia of NMR (Ref. 8), p. 2932.

${ }^{13}$ J. W. Emsley and M. Longeri, Mol. Phys. 42, 315 (1981).

${ }^{14}$ T. C. Wong and E. E. Burnell, J. Magn. Reson. (1969-1992) 22, 227 (1976).

${ }^{15}$ J. W. Emsley and G. R. Luckhurst, Mol. Phys. 41, 19 (1980).

${ }^{16}$ S. A. Ramadan, L. D. Field, and G. K. Pierens, Mol. Phys. 101, 1813 (2003).

${ }^{17}$ G. Celebre, G. De Luca, M. Longeri, and E. Sicilia, J. Chem. Inf. Comput. Sci. 34, 539 (1994).

${ }^{18}$ W. Brugel, NMR Spectra and Chemical Structure (Academic, New York, 1967).

${ }^{19}$ H. O. Kalinowski, S. Berger, and S. Braun, Carbon 13 NMR Spectroscopy (Wiley, New York, 1996). 
${ }^{20}$ S. Sýkora, J. Vogt, H. Bösiger, and P. Diehl, J. Magn. Reson. (1969-1992) 36, 53 (1979).

${ }^{21}$ G. De Luca, M. Longeri, and G. Pileio (unpublished).

${ }^{22}$ D. A. Condirston and J. D. Laposa, J. Mol. Spectrosc. 63, 466 (1976).

${ }^{23}$ J. Lounila and P. Diehl, Mol. Phys. 52, 827 (1984).

${ }^{24}$ R. T. Syvitski and E. E. Burnell, J. Magn. Reson. (1969-1992) 144, 58 (2000).
${ }^{25}$ J. W. Emsley and J. C. Lindon, NMR Spectroscopy Using Liquid Crystalline Solvents (Pergamon, Oxford, 1975).

${ }^{26}$ J. W. Emsley, G. R. Luckhurst, and C. P. Stockley, Proc. R. Soc. London, Ser. A 381, 117 (1982).

${ }^{27}$ G. Celebre, G. De Luca, J. W. Emsley, E. K. Foord, M. Longeri, F. Lucchesini, and G. Pileio, J. Chem. Phys. 118, 6417 (2003). 